\title{
ON FINITE SOLUBLE GROUPS WITH ALMOST FIXED-POINT-FREE AUTOMORPHISMS OF NON-COPRIME ORDER
}

\author{
E. I. KHUKHRO
}

\begin{abstract}
It is proved that if a finite $p$-soluble group $G$ admits an automorphism $\varphi$ of order $p^{n}$ having at most $m$ fixed points on every $\varphi$-invariant elementary abelian $p^{\prime}$-section of $G$, then the $p$-length of $G$ is bounded above in terms of $p^{n}$ and $m$; if in addition the group $G$ is soluble, then the Fitting height of $G$ is bounded above in terms of $p^{n}$ and $m$. It is also proved that if a finite soluble group $G$ admits an automorphism $\psi$ of order $p^{a} q^{b}$ for some primes $p, q$, then the Fitting height of $G$ is bounded above in terms of $|\psi|$ and $\left|C_{G}(\psi)\right|$.
\end{abstract}

to Yurii Leonidovich Ershov on the occasion of his 75-th birthday

\section{INTRODUCTION}

Studying groups with "almost fixed-point-free" automorphisms means obtaining restrictions on the structure of groups depending on their automorphisms and certain restrictions imposed on the fixed-point subgroups. In this paper we consider questions of bounding the $p$-length and Fitting height of finite $p$-soluble and soluble groups admitting almost fixed-point-free automorphisms of non-coprime order.

Let $\varphi \in$ Aut $G$ be an automorphism of a finite group $G$. Studying the structure of the group $G$ depending on $\varphi$ and the fixed-point subgroup $C_{G}(\varphi)$ is one of the most important and fruitful avenues in finite group theory. The celebrated Brauer-Fowler theorem [1] (bounding the index of the soluble radical in terms of the order of $\left|C_{G}(\varphi)\right|$ when $|\varphi|=2$ ) and Thompson's theorem [2] (giving the nilpotency of $G$ when $\varphi$ is of prime order and acts fixed-point-freely, that is, $C_{G}(\varphi)=1$ ) lie in the foundations of the classification of finite simple groups. The classification was used for obtaining further results on solubility of $G$, or of a suitable "large" subgroup. For example, using the classification Hartley [3] generalized the Brauer-Fowler theorem to any order of $\varphi$ : the group $G$ has a soluble subgroup of index bounded in terms of $|\varphi|$ and $\left|C_{G}(\varphi)\right|$.

Now suppose that the group $G$ is soluble. Further information on the structure of $G$ is sought first of all in the form of bounds for the Fitting height (nilpotent length). A bound for the Fitting height naturally reduces further studies to the case of nilpotent groups with (almost) fixed-point-free automorphisms, for which, in turn, problems arise of bounding the derived length, or the nilpotency class of the group or of a suitable "large" subgroup. Such bounds for nilpotent groups so far have been obtained in the cases of $\varphi$ being of prime order or of order 4 in $[4,5,6,7,8,9]$. In addition, definitive general results have been obtained in the study of almost fixed-point-free $p$-automorphisms of finite $p$-groups $[10,11,12,13,14,15]$.

On bounding the Fitting height, especially strong results have been obtained in the case of soluble groups of automorphisms $A \leqslant$ Aut $G$ of coprime order. Thompson [16] proved that if both groups $G$ and $A$ are soluble and have coprime orders, then the Fitting height

Key words and phrases. finite soluble group, automorphism, p-length, Fitting height.

The work is supported by Russian Science Foundation (project 14-21-00065). 
of $G$ is bounded in terms of the Fitting height of $C_{G}(A)$ and the number $\alpha(A)$ of prime factors of $|A|$ with account for multiplicities. Later the bounds in Thompson's theorem were improved in numerous papers, with definitive results obtained by Turull [17] and Hartley and Isaacs [18] with linear bounds in terms of $\alpha(A)$ for the Fitting height of the group or of a "large subgroup".

The case of non-coprime orders of $G$ and $A \leqslant$ Aut $G$ is more difficult. Bell and Hartley [19] constructed examples showing that for any non-nilpotent finite group $A$ there are soluble groups $G$ of arbitrarily high Fitting height admitting $A$ as a fixed-point-free group of automorphisms. But if $A$ is nilpotent and $C_{G}(A)=1$, then the Fitting height of $G$ is bounded in terms of $\alpha(A)$ by a special case of Dade's theorem [20]. Unlike the aforementioned "linear" results in the coprime case, the bound in Dade's theorem is exponential. Improving this bound to a linear one is a difficult problem; it was tackled in some special cases by Ercan and Güloğlu [21, 22, 23].

In the almost fixed-point-free situation, even for a cyclic group of automorphisms $\langle\varphi\rangle \leqslant$ Aut $G$ it is still an open problem to obtain a bound for the Fitting height of a finite soluble group $G$ in terms of $|\varphi|$ and $\left|C_{G}(\varphi)\right|$ (this question is equivalent to the one recorded by Belyaev in Kourovka Notebook [24] as Hartley's Problem 13.8(a)). Beyond the fixedpoint-free case of Dade's theorem, so far the only cases where an affirmative solution is known are the cases of automorphisms of primary order $p^{n}$ (Hartley and Turau [25]) and of biprimary order $p^{a} q^{b}$ (which is discussed in the present paper).

Another generalization of fixed-point-free automorphisms in the non-coprime case is Thompson's problem on bounding the $p$-length of a finite $p$-soluble group $G$ admitting a $p$-group of automorphisms $P$ that acts fixed-point-freely on every $P$-invariant $p^{\prime}$-section of $G$. Rae [26] and Hartley and Rae [27] solved this problem in the affirmative for $p \neq 2$, as well as for cyclic $P$ for any $p$. A special case of this problem is when a $p$-soluble group $G$ admits a so-called $p^{n}$-splitting automorphism $\varphi$, which means that $x x^{\varphi} x^{\varphi^{2}} \cdots x^{\varphi^{p^{n}-1}}=1$ for all $x \in G$ (this also implies $\varphi^{p^{n}}=1$ ); then of course $\varphi$ automatically acts fixed-pointfreely on $\varphi$-invariant $p^{\prime}$-sections. This case was actually considered earlier by Kurzweil [28] who obtained bounds for the Fitting height of a soluble group $G$, and these bounds were improved to linear ones by Meixner [29]. If it is only known that $\varphi$ induces a $p^{n}$-splitting automorphism on a $\varphi$-invariant Sylow $p$-subgroup of $G$, then there is already a bound in terms of $n$ for the $p$-length of $G$ : for $p \neq 2$ such a bound was obtained by Wilson [30], and for all primes $p$ in [31] even under a weaker assumption.

In this paper we consider the natural generalization of Thompson's problem for a $p$ soluble group $G$ admitting an automorphism $\varphi$ of order $p^{n}$ in which the condition that $\varphi$ acts fixed-point-freely on $\varphi$-invariant $p^{\prime}$-sections is replaced by that $\varphi$ acts almost fixedpoint-freely on these sections. It is actually sufficient to impose the restriction on the number of fixed points of $\varphi$ only on elementary abelian $\varphi$-invariant $p^{\prime}$-sections.

Theorem 1.1. If a finite $p$-soluble group $G$ admits an automorphism $\varphi$ of order $p^{n}$ such that $\varphi$ has at most $m$ fixed points on every $\varphi$-invariant elementary abelian $p^{\prime}$-section of $G$, then the p-length of $G$ is bounded above in terms of $p^{n}$ and $m$.

It would be interesting to obtain a bound of the $p$-length in terms of $n$ (or at least in terms of $p^{n}$ ) for some subgroup of index bounded in terms of $p^{n}$ and $m$.

Remark 1.2. There is a certain similarity with the situation for a $p^{n}$-splitting automorphism described above. Namely, if, for a $p$-soluble group $G$ with an automorphism $\varphi$ of order $p^{n}$, instead of a restriction on the number of fixed points on $p^{\prime}$-sections, we have a restriction $\left|C_{P}(\varphi)\right|=p^{m}$ on the number of fixed points of $\varphi$ in a $\varphi$-invariant Sylow $p$ subgroup $P$, then we also obtain a bound for the $p$-length of $G$. Indeed, then the derived 
length of $P$ is bounded in terms of $p, n$, and $m$ by Shalev's theorem [12], so the bound for the $p$-length immediately follows from the Hall-Higman theorems [32] for $p \neq 2$, and the theorems of Hoare [33], Berger and Gross [34], and Bryukhanova [35]. Moreover, by [13] the group $P$ even has a (normal) subgroup of index bounded in terms of $p, n$, and $m$ that has $p^{n}$-bounded derived length. Therefore by the Hall-Higman-Hartley Theorem 2.3 (see below) there is a characteristic subgroup $H$ of $G$ such that the $p$-length of $H$ is $p^{n}$-bounded and a Sylow $p$-subgroup of the quotient $G / H$ has order bounded in terms of $p, n$, and $m$.

For soluble groups, Theorem 1.1 can be combined with known results to give a bound for the Fitting height.

Corollary 1.3. If a finite soluble group $G$ admits an automorphism $\varphi$ of order $p^{n}$ such that $\varphi$ has at most $m$ fixed points on every $\varphi$-invariant elementary abelian $p^{\prime}$-section of $G$, then the Fitting height of $G$ is bounded above in terms of $p^{n}$ and $m$.

The technique used in the proof of Theorem 1.1 is also applied in the proof of the soluble case of the following theorem on almost fixed-point-free automorphism of biprimary order; the reduction to the soluble case is given by Hartley' theorem [3] (based on the classification of finite simple groups).

Theorem 1.4. If a finite group $G$ admits an automorphism $\varphi$ of order $p^{a} q^{b}$ for some primes $p, q$ and nonnegative integers $a, b$, then $G$ has a soluble subgroup whose index and Fitting height are bounded above in terms of $p^{a} q^{b}$ and $\left|C_{G}(\varphi)\right|$.

Standard inverse limit arguments yield the following corollary for locally finite groups.

Corollary 1.5. If a locally finite group $G$ contains an element $g$ of order $p^{a} q^{b}$ for some primes $p, q$ and nonnegative integers $a, b$ with finite centralizer $C_{G}(g)$, then $G$ has a subgroup of finite index that has a finite normal series with locally nilpotent factors.

Another corollary is of more technical nature but it may be useful in further studies.

Corollary 1.6. If a finite group $G$ admits an automorphism $\varphi$ such that there are at most two primes dividing both $|\varphi|$ and $|G|$, then $G$ has a soluble subgroup whose index and Fitting height are bounded above in terms of $|\varphi|$ and $\left|C_{G}(\varphi)\right|$.

Remark 1.7. After this paper was prepared for publication, the author became aware of an unpublished manuscript of Brian Hartley, which contains the result of Theorem 1.4; the author together with A. Borovik and P. Shumyatsky published this manuscript as [36] on the web-site of the University of Manchester.

\section{Preliminaries}

Induced automorphisms of invariant sections are denoted by the same letters. The following lemma is well known.

Lemma 2.1. If $\varphi$ is an automorphism of a finite group $G$ and $N$ is a normal $\varphi$-invariant subgroup, then $\left|C_{G / N}(\varphi)\right| \leqslant\left|C_{G}(\varphi)\right|$.

The next lemma is also a well-known consequence of considering the Jordan normal form of a linear transformation of order $p^{k}$ in characteristic $p$.

Lemma 2.2. If an elementary abelian $p$-group $P$ admits an automorphism $\varphi$ of order $p^{k}$ such that $\left|C_{P}(\varphi)\right|=p^{m}$, then the rank of $P$ is bounded in terms of $p^{k}$ and $m$.

We shall use the following consequence of the Hall-Higman-type theorems in Hartley's paper [37]. 
Theorem 2.3 (Hall-Higman-Hartley). Let $P$ be a Sylow p-subgroup of a p-soluble group $G$. If $R$ is a normal subgroup of $P$ and the derived length of $R$ is $d$, then $R \leqslant O_{p^{\prime}, p, p^{\prime}, \ldots, p^{\prime}, p}(G)$, where $p$ occurs on the right-hand side $d$ times if $p>3,2 d$ times if $p=3$, and $3 d$ times if $p=2$.

Proof. As a refinement of some of the Hall-Higman theorems [32], Hartley [37] proved that if $A$ is an abelian normal subgroup of a Sylow $p$-subgroup of $G$, then

$$
\begin{gathered}
A \leqslant O_{p^{\prime}, p}(G) \quad \text { if } \quad p>3, \\
A \leqslant O_{3^{\prime}, 3,3^{\prime}, 3}(G) \quad \text { if } \quad p=3,
\end{gathered}
$$

and

$$
A \leqslant O_{2^{\prime}, 2,2^{\prime}, 2,2^{\prime} 2}(G) \quad \text { if } \quad p=2 .
$$

The result follows from these inclusions for $A=R^{(d-1)}$ by a straightforward induction on the derived length $d$.

We now recall some definitions and notation from representation theory. If $V$ is a $k G$ module for a field $k$ and a group $G$, we use the right operator notation $v g$ for $v \in V$ and $g \in G$. We use the centralizer notation for fixed points, like $C_{V}(g)=\{v \in V \mid v g=v\}$. We also use the commutator notation $[v, g]=-v+v g$ for $v \in V$ and $g \in G$. The commutator subspaces are defined accordingly: if $B \leqslant G$, then $[V, B]$ is the span of all commutators $[v, b]$, where $v \in V$ and $b \in B$. The subspace $[V, B]$ coincides with the commutator subgroup $[V, B]$ in the natural semidirect product $V G$ when $V$ is regarded as the additive group acted upon by $G$. In particular, $[V, B]$ is $B$-invariant, and thus can be regarded as a $k B$-submodule.

For a group $G$ and a field $k$, a free $k G$-module of rank $n$ is a direct sum of $n$ copies of the group algebra $k G$ each of which is regarded as a vector space over $k$ of dimension $|G|$ with a basis $\left\{b_{g} \mid g \in G\right\}$ labelled by elements of $G$ on which $G$ acts in a regular permutation representation: $b_{g} h=b_{g h}$. In other words, a free $k G$-module $V=\bigoplus_{g \in G} V_{g}$ is a direct sum of subspaces that are regularly permuted by $G$ so that $V_{g} h=V_{g h}$.

The following lemma is known in the literature (see, for example, [25, Lemma 4.5]), but we give a proof for completeness.

Lemma 2.4. Suppose that an abelian p-group $M$ is acted upon by a cyclic group $\langle\varphi\rangle$ of order $p^{n}$ and $V$ is a $k M\langle\varphi\rangle$-module for a field $k$ of characteristic different from $p$. If the subgroup $\left[M, \varphi^{p^{n-1}}\right]$ acts non-trivially on $V$, then the subspace $\left[V,\left[M, \varphi^{p^{n-1}}\right]\right]$ is a free $k\langle\varphi\rangle$-module.

Here, of course, $\varphi^{p^{n-1}}=\varphi$ if $n=1$.

Proof. The subspace $\left[V,\left[M, \varphi^{p^{n-1}}\right]\right]$ is clearly $M\langle\varphi\rangle$-invariant, so is an $k M\langle\varphi\rangle$-module. We extend the ground field to its algebraic closure $\bar{k}$ and denote by $W=V \otimes_{k} \bar{k}$ the resulting $\bar{k} M\langle\varphi\rangle$-module. Then $\left[W,\left[M, \varphi^{p^{n-1}}\right]\right]$ is a $\bar{k} M\langle\varphi\rangle$-module obtained from $\left[V,\left[M, \varphi^{p^{n-1}}\right]\right]$ by the field extension.

Since the characteristic of the ground field is coprime to $|M\langle\varphi\rangle|$, by Maschke's theorem

$$
W=C_{W}\left(\left[M, \varphi^{p^{n-1}}\right]\right) \oplus\left[W,\left[M, \varphi^{p^{n-1}}\right]\right]
$$

is a completely reducible $\bar{k} M\langle\varphi\rangle$-module. Let $U$ be an irreducible $\bar{k} M\langle\varphi\rangle$-submodule of $\left[W,\left[M, \varphi^{p^{n-1}}\right]\right]$ on which $\left[M, \varphi^{p^{n-1}}\right]$ acts non-trivially.

By Clifford's theorem, $U=U_{1} \oplus \cdots \oplus U_{m}$ decomposes into homogeneous $\bar{k} M$-submodules $U_{i}$ (Wedderburn components). The group $\langle\varphi\rangle$ transitively permutes the $U_{i}$. If the kernel of this permutational action was non-trivial, then $\varphi^{p^{n-1}}$ would stabilize all the $U_{i}$. But 
the abelian group $M$ acts by scalar transformations on each homogeneous component $U_{i}$. Hence $\left[M, \varphi^{p^{n-1}}\right]$ would act trivially on each $U_{i}$ and therefore on $U$, contrary to our assumption. Thus, $U$ is a free $\bar{k}\langle\varphi\rangle$-module.

Since $\left[W,\left[M, \varphi^{p^{n-1}}\right]\right]$ is the direct sum of such $U$, we obtain that $\left[W,\left[M, \varphi^{p^{n-1}}\right]\right]$ is also a free $\bar{k}\langle\varphi\rangle$-module. Then $\left[V,\left[M, \varphi^{p^{n-1}}\right]\right]$ is a free $k\langle\varphi\rangle$-module. Indeed, by the DeuringNoether theorem [38, Theorem 29.7] two representations over a smaller field are equivalent if they are equivalent over a larger field. Being a free $\bar{k}\langle\varphi\rangle$-module, or a free $k\langle\varphi\rangle$-module, means having a basis, as of a vector space over the corresponding field, elements of which are permuted by $\varphi$ so that all orbits are regular. In such a basis $\langle\varphi\rangle$ is represented by the corresponding permutational matrices, all of which are defined over $k$.

\section{Automorphism of order $p^{n}$}

First we state separately the following proposition, which will also be used in the next section in a different situation.

Proposition 3.1. Suppose that a cyclic group $\langle\varphi\rangle$ of order $p^{n}$ acts by automorphisms on a finite p-group $P$, and $V$ is a faithful $\mathbb{F}_{q} P\langle\varphi\rangle$-module, where $\mathbb{F}_{q}$ is a prime field of order $q \neq p$. Then the derived length of $\left[P, \varphi^{p^{n-1}}\right]$ is bounded in terms of $\left|C_{V}(\varphi)\right|$ and $p^{n}$.

Proof. Let $M$ be a maximal abelian normal subgroup of the semidirect product $P\langle\varphi\rangle$. If $\left[M, \varphi^{p^{n-1}}\right] \neq 1$, then by Lemma $2.4,\left[V,\left[M, \varphi^{p^{n-1}}\right]\right]$ is a free $\mathbb{F}_{q}\langle\varphi\rangle$-module. Obviously, in a free $\mathbb{F}_{q}\langle\varphi\rangle$-module the fixed points of $\varphi$ are exactly the "diagonal" elements. Hence the order of $\left[V,\left[M, \varphi^{p^{n-1}}\right]\right]$ is equal to

$$
\left|C_{\left[V,\left[M, \varphi^{p^{n-1}}\right]\right]}(\varphi)\right|^{|\varphi|}=\left|C_{\left[V,\left[M, \varphi^{p^{n-1}}\right]\right]}(\varphi)\right|^{p^{n}}
$$

and therefore is bounded in terms of $\left|C_{V}(\varphi)\right|$ and $p^{n}$. The group $\left[M, \varphi^{p^{n-1}}\right]$ acts faithfully on $V$; therefore by Maschke's theorem it also acts faithfully on $\left[V,\left[M, \varphi^{p^{n-1}}\right]\right]$. Hence the order of $\left[M, \varphi^{p^{n-1}}\right]$ is bounded in terms of $\left|C_{V}(\varphi)\right|$ and $p^{n}$. The same of course holds if $\left[M, \varphi^{p^{n-1}}\right]=1$.

It follows that the index $\left|M: C_{M}\left(\varphi^{p^{n-1}}\right)\right|$ is bounded in terms of $\left|C_{V}(\varphi)\right|$ and $p^{n}$, since this index is equal to the number of different commutators $\left[m, \varphi^{p^{n-1}}\right]$ for $m \in M$.

Consider a central series of $P\langle\varphi\rangle$ connecting 1 and $M$. Since $\left|M: C_{M}\left(\varphi^{p^{n-1}}\right)\right|$ is bounded in terms of $\left|C_{V}(\varphi)\right|$ and $p^{n}$, the number of factors of this series that are not covered by $C_{M}\left(\varphi^{p^{n-1}}\right)$ is bounded in terms of $\left|C_{V}(\varphi)\right|$ and $p^{n}$. Therefore there is a normal series of bounded length connecting 1 and $M$ each factor of which is either central in $P\langle\varphi\rangle$ or is covered by $C_{M}\left(\varphi^{p^{n-1}}\right)$. Obviously, then $\varphi^{p^{n-1}}$ acts trivially on each factor of this series, and therefore so does $\left[P, \varphi^{p^{n-1}}\right]$. By Kaluzhnin's theorem, the automorphism group induced by the action of $\left[P, \varphi^{p^{a-1}}\right]$ on $M$ is nilpotent of bounded class. Since $M$ contains its centralizer in $P\langle\varphi\rangle$, it follows that $\left[P, \varphi^{p^{n-1}}\right]$ is soluble of bounded derived length, since by the above $\gamma_{s}\left(\left[P, \varphi^{p^{n-1}}\right]\right) \leqslant M \cap\left[P, \varphi^{p^{n-1}}\right]$ for some number $s$ bounded in terms of $\left|C_{V}(\varphi)\right|$ and $p^{n}$.

Proof of Theorem 1.1. Recall that $G$ is a finite $p$-soluble group admitting an automorphism $\varphi$ of order $p^{n}$ such that $\varphi$ has at most $m$ fixed points in every $\varphi$-invariant elementary abelian $p^{\prime}$-section of $G$. We need to bound the $p$-length of $G$ in terms of $p^{n}$ and $m$. Henceforth in this section, saying for brevity that a certain parameter is simply "bounded" we mean that this parameter is bounded above in terms of $p^{n}$ and $m$.

We use induction on $n$. It is convenient to consider the case of $n=0$ as the basis of induction, when $|\varphi|=p^{0}=1$, that is, $\varphi$ acts trivially on $G$. Then the hypothesis means 
that every elementary abelian $p^{\prime}$-section of $G$ has bounded order. We claim that the nilpotency class of a Sylow $p$-subgroup $P$ of $\hat{G}=G / O_{p}(G)$ is bounded. Indeed, since the order of $P$ is coprime to $\left|O_{p^{\prime}}(\hat{G})\right|$, for every prime $q$ dividing $\left|O_{p^{\prime}}(\hat{G})\right|$ there is a $P$-invariant Sylow $q$-subgroup $Q$ of $O_{p^{\prime}}(\hat{G})$. The quotient $P / C_{P}(Q)$ acts faithfully on the Frattini quotient $Q / \Phi(Q)$, which has order at most $m$ by the assumption. Hence $P / C_{P}(Q)$ has bounded order and therefore bounded nilpotency class. Since $P$ acts faithfully on $O_{p^{\prime}}(\hat{G})$, we have $\bigcap C_{P}\left(Q_{i}\right)=1$, where $Q_{i}$ runs over all $P$-invariant Sylow subgroups of $O_{p^{\prime}}(\hat{G})$. Hence $P$ is a subdirect product of groups of bounded nilpotency class and therefore has bounded nilpotency class itself. We now obtain that the $p$-length of $\hat{G}=G / O_{p}(G)$ is bounded by the Hall-Higman theorem [32]. As a result, the $p$-length of $G$ is bounded.

From now on we assume that $n \geqslant 1$.

Let $\hat{G}=G / O_{p}(G)$. Consider a Sylow $p$-subgroup of the semidirect product $\hat{G}\langle\varphi\rangle$ containing $\langle\varphi\rangle$ and let $P$ be its intersection with $\hat{G}$, so that $P$ is a $\varphi$-invariant Sylow $p$-subgroup of $\hat{G}$. Since the order of the p-group $P\langle\varphi\rangle$ is coprime to $\left|O_{p^{\prime}}(\hat{G})\right|$, for every prime $q$ dividing $\left|O_{p^{\prime}}(\hat{G})\right|$ there is a $P\langle\varphi\rangle$-invariant Sylow $q$-subgroup $Q$ of $O_{p^{\prime}}(\hat{G})$.

The quotient $\bar{P}=P / C_{P}(Q)$ acts faithfully on the Frattini quotient $V=Q / \Phi(Q)$, which we regard as an $\mathbb{F}_{q} P\langle\varphi\rangle$-module. By hypothesis, $\left|C_{V}(\varphi)\right| \leqslant m$, so by Proposition 3.1 the derived length of $\left[\bar{P}, \varphi^{p^{n-1}}\right]$ is bounded. In other words, $\left[P, \varphi^{p^{n-1}}\right]^{(s)} \leqslant C_{P}(Q)$ for some bounded number $s$. Since $P$ acts faithfully on $O_{p^{\prime}}(\hat{G})$, we have $\bigcap C_{P}\left(Q_{i}\right)=1$, where $Q_{i}$ runs over all $P\langle\varphi\rangle$-invariant Sylow subgroups of $O_{p^{\prime}}(\hat{G})$. Hence, $\left[P, \varphi^{p^{n-1}}\right]^{(s)}=1$.

By the Hall-Higman-Hartley Theorem 2.3 we now obtain that the normal subgroup $\left[P, \varphi^{p^{n-1}}\right]$ of the Sylow $p$-subgroup $P$ is contained in $H=O_{p^{\prime}, p, p^{\prime}, \ldots, p^{\prime}, p}(\hat{G})$, where $p$ occurs boundedly many times.

Consider the action of $\varphi$ on the quotient $\tilde{G}=\hat{G} / H$. Since $\left[P, \varphi^{p^{n-1}}\right] \leqslant H$, it follows that $\varphi^{p^{n-1}}$ acts trivially on the image of $P$, which is a Sylow $p$-subgroup of $\tilde{G}$. In particular, $\varphi^{p^{n-1}}$ acts trivially on $O_{p^{\prime}, p}(\tilde{G}) / O_{p^{\prime}}(\tilde{G})$, and therefore so does $\left[\tilde{G}, \varphi^{p^{n-1}}\right]$. Since $O_{p^{\prime}, p}(\tilde{G}) / O_{p^{\prime}}(\tilde{G})$ contains its centralizer in $\tilde{G} / O_{p^{\prime}}(\tilde{G})$, we obtain that $\left[\tilde{G}, \varphi^{p^{n-1}}\right] \leqslant O_{p^{\prime}, p}(\tilde{G})$. In other words, $\varphi^{p^{n-1}}$ acts trivially on the quotient $\tilde{G} / O_{p^{\prime}, p}(\tilde{G})$. Therefore the order of the automorphism induced by $\varphi$ on $\tilde{G} / O_{p^{\prime}, p}(\tilde{G})$ is at most $p^{n-1}$. By the induction hypothesis the $p$-length of this quotient is bounded. Then the $p$-length of $G / O_{p, p^{\prime}}(G)$ is bounded, and therefore the $p$-length of $G$ is bounded, as required.

Proof of Corollary 1.3. Here, $G$ is a finite soluble group admitting an automorphism $\varphi$ of order $p^{n}$ such that $\varphi$ has at most $m$ fixed points in every $\varphi$-invariant elementary abelian $p^{\prime}$-section of $G$. By Theorem 1.1 the $p$-length of $G$ is bounded. It remains to obtain a bound for the Fitting height of every $p^{\prime}$-factor $T$ of the upper $p$-series consisting of the subgroups $O_{p^{\prime}, p, p^{\prime}, p, \ldots}$. It is known that the rank of a finite group is bounded in terms of the ranks of its elementary abelian sections. Here, by definition, the rank of a group is the minimum number $r$ such that every subgroup can be generated by $r$ elements. Of course every elementary abelian section of $C_{T}(\varphi)$ is a $\varphi$-invariant $p^{\prime}$-section of $G$ and therefore has bounded order by hypothesis. It is also known that the Fitting height of a soluble finite group is bounded in terms of its rank. Thus $C_{T}(\varphi)$ has bounded Fitting height and therefore so does $G$ by Thompson's theorem [16].

Remark 3.2. If we could obtain in Theorem 1.1 a "strong" bound for the $p$-length, in terms of $\alpha(\langle\varphi\rangle)$ only, for a subgroup of bounded index, then a similar strong bound could be obtained in Corollary 1.3 for the Fitting height of a subgroup of bounded index. This would follow from a rank analogue of the Hartley-Isaacs theorem proved in [39], which 
states that if a finite soluble group $K$ admits a soluble group of automorphisms $L$ of coprime order, then $K$ has a normal subgroup $N$ of Fitting height at most $5\left(4^{\alpha(L)}-1\right) / 3$ such that the order of $K / N$ is bounded in terms of $|L|$ and the rank of $C_{K}(L)$.

\section{Automorphism of ORDER $\boldsymbol{p}^{\boldsymbol{a}} \boldsymbol{q}^{\boldsymbol{b}}$}

Proof of Theorem 1.4. Recall that $G$ is a finite group admitting an automorphism $\varphi$ of order $p^{a} q^{b}$. By Hartley's theorem [3] (based on the classification of finite simple groups), $G$ has a soluble subgroup of index bounded in terms of $p^{a} q^{b}$ and $\left|C_{G}(\varphi)\right|$. Therefore we can assume from the outset that $G$ is soluble, so that we need to bound the Fitting height of $G$ in terms of $p^{a} q^{b}$ and $\left|C_{G}(\varphi)\right|$. Throughout this section we say for brevity that a certain parameter is "bounded" meaning that this parameter is bounded above in terms of $p^{a} q^{b}$ and $\left|C_{G}(\varphi)\right|$. We use without special references the fact that the number of fixed points of $\varphi$ in every $\varphi$-invariant section of $G$ is at most $\left|C_{G}(\varphi)\right|$ by Lemma 2.1.

We use induction on $a+b$. As a basis of induction we consider the case when either $a=0$ or $b=0$. Then $|\varphi|$ is a prime-power, and by the Hartley-Turau theorem [25] the group $G$ has a subgroup of bounded index that has Fitting height at most $\alpha(\varphi)$. (Actually, for our 'weak' bound a simpler argument would suffice: if, say, $|\varphi|=p^{a}$, then the rank of the Frattini quotient of $O_{p^{\prime}, p}(G) / O_{p^{\prime}}(G)$ is bounded by Lemma 2.2, which implies a bound for the Fitting height of $G / O_{p^{\prime}}(G)$, and the Fitting height of $O_{p^{\prime}}(G)$ is bounded in terms of $a$ by Thompson's theorem [16].) Moreover, the following proposition holds, which apparently was noted by Hartley but may have remained unpublished. We state this proposition in a more general form, without assuming that the automorphism has biprimary order.

Proposition 4.1. If a finite soluble group $G$ admits an automorphism $\psi$ such that there is at most one prime dividing both $|\psi|$ and $|G|$, then the Fitting height of $G$ is bounded above in terms of $|\psi|$ and $\left|C_{G}(\psi)\right|$.

Proof. If $(|\psi|,|G|)=1$, then the result follows from the stronger theorem of Thompson [16]. Now let $\langle\psi\rangle=\left\langle\psi_{r}\right\rangle \times\left\langle\psi_{r^{\prime}}\right\rangle$, where $\left\langle\psi_{r}\right\rangle$ is the Sylow $r$-subgroup of $\langle\psi\rangle$ and $r$ is the only common prime divisor of $|G|$ and $|\psi|$. The centralizer $C_{G}\left(\psi_{r^{\prime}}\right)$ admits the automorphism $\psi_{r}$ of prime-power order whose centralizer $C_{C_{G}\left(\psi_{r^{\prime}}\right)}\left(\psi_{r}\right)$ is equal to $C_{G}(\psi)$. By the Hartley-Turau theorem, the Fitting height of $C_{G}\left(\psi_{r^{\prime}}\right)$ is bounded. We now apply Thompson's theorem to the automorphism $\psi_{r^{\prime}}$ of $G$ of coprime order to obtain that the Fitting height of $G$ is bounded as required.

We return to the proof of Theorem 1.4. Let $a \geqslant 1$ and $b \geqslant 1$. Let $\varphi_{p}=\varphi^{q^{b}}$ and $\varphi_{q}=\varphi^{p^{a}}$, so that $\left|\varphi_{p}\right|=p^{a}$ and $\left|\varphi_{q}\right|=q^{b}$, while $\langle\varphi\rangle=\left\langle\varphi_{p}\right\rangle \times\left\langle\varphi_{q}\right\rangle$. The subgroup $O_{q^{\prime}}(G)$ admits the automorphism $\varphi$ whose order has at most one prime divisor $p$ in common with $\left|O_{q^{\prime}}(G)\right|$. By Proposition 4.1 the Fitting height of $O_{q^{\prime}}(G)$ is bounded.

Therefore we can assume that $O_{q^{\prime}}(G)=1$. Then the quotient $\bar{G}=G / O_{q}(G)$ acts faithfully on the Frattini quotient $V=O_{q}(G) / \Phi\left(O_{q}(G)\right)$, which we regard as an $\mathbb{F}_{q} \bar{G}\langle\varphi\rangle$ module. The fixed-point subspace $C_{V}\left(\varphi_{p}\right)$ has bounded order. This follows from Lemma 2.2 applied to the action of the linear transformation $\varphi_{q}$ of order $q^{b}$ on $C_{V}\left(\varphi_{p}\right)$, since the fixed points of $\varphi_{q}$ in $C_{V}\left(\varphi_{p}\right)$ are contained in the fixed-point subspace $C_{V}(\varphi)$ of bounded order.

Choose a Sylow $p$-subgroup of $\bar{G}\left\langle\varphi_{p}\right\rangle$ containing $\left\langle\varphi_{p}\right\rangle$, and let $P$ be its intersection with $\bar{G}$, so that $P$ is a $\varphi_{p}$-invariant Sylow $p$-subgroup of $\bar{G}$. By Proposition 3.1, the subgroup $\left[P, \varphi_{p}^{p^{a-1}}\right]$ has bounded derived length. 
Hence by the Hall-Higman-Hartley Theorem 2.3 the normal subgroup $\left[P, \varphi_{p}^{p^{a-1}}\right]$ of the Sylow $p$-subgroup $P$ is contained in $H=O_{p^{\prime}, p, p^{\prime}, \ldots, p^{\prime}, p}(\bar{G})$, where $p$ occurs boundedly many times.

Consider the action of $\varphi$ on the quotient $\tilde{G}=\bar{G} / H$. Since $\left[P, \varphi_{p}^{p^{a-1}}\right] \leqslant H$, it follows that $\varphi_{p}^{p^{a-1}}$ acts trivially on the image of $P$, which is a Sylow $p$-subgroup of $\tilde{G}$. In particular, $\varphi_{p}^{p^{a-1}}$ acts trivially on $O_{p^{\prime}, p}(\tilde{G}) / O_{p^{\prime}}(\tilde{G})$, and therefore so does $\left[\tilde{G}, \varphi_{p}^{p^{a-1}}\right]$. Since $O_{p^{\prime}, p}(\tilde{G}) / O_{p^{\prime}}(\tilde{G})$ contains its centralizer in $\tilde{G} / O_{p^{\prime}}(\tilde{G})$, we obtain that $\left[\tilde{G}, \varphi_{p}^{p^{a-1}}\right] \leqslant O_{p^{\prime}, p}(\tilde{G})$. In other words, $\varphi_{p}^{p^{a-1}}$ acts trivially on the quotient $\tilde{G} / O_{p^{\prime}, p}(\tilde{G})$. Therefore the order of the automorphism induced by $\varphi$ on $\tilde{G} / O_{p^{\prime}, p}(\tilde{G})$ divides $p^{a-1} q^{b}$. By induction, the Fitting height of this quotient is bounded.

It remains to obtain a bound for the Fitting height of each of the boundedly many $\varphi$-invariant normal $p^{\prime}$-sections that appear in the upper $p$-series of the groups $H$ and $O_{p^{\prime}, p}(\tilde{G})$. Such a bound follows from Proposition 4.1.

Proof of Corollary 1.5. This corollary for locally finite groups follows from Theorem 1.4 by the standard inverse limit argument.

Proof of Corollary 1.6. Here, a finite group $G$ admits an automorphism $\varphi$ such that there are at most two primes dividing both $|\varphi|$ and $|G|$. Again, by Hartley's theorem [3] we can assume from the outset that $G$ is soluble. If $(|\varphi|,|G|)$ is 1 or a prime power, then the result follows from Proposition 4.1. Now let $\langle\varphi\rangle=\left\langle\varphi_{p q}\right\rangle \times\langle\psi\rangle$, where $\left\langle\varphi_{p q}\right\rangle$ is the Hall $\{p, q\}$-subgroup of $\langle\varphi\rangle$ and $p, q$ are the only common prime divisors of $|G|$ and $|\varphi|$. The centralizer $C_{G}(\psi)$ admits the automorphism $\varphi_{p q}$ of biprimary order whose centralizer $C_{C_{G}(\psi)}\left(\varphi_{p q}\right)$ is equal to $C_{G}(\varphi)$. By Theorem 1.4, the Fitting height of $C_{G}(\psi)$ is bounded in terms of $\left|\varphi_{p q}\right|$ and $\left|C_{G}(\varphi)\right|$. We now apply Thompson's theorem [16] to the automorphism $\psi$ of $G$ of coprime order to obtain that the Fitting height of $G$ is bounded in terms of $|\varphi|$ and $\left|C_{G}(\varphi)\right|$.

\section{REFERENCES}

[1] R. Brauer and K. A. Fowler, On groups of even order, Ann. Math. (2) 62 (1955), 565-583.

[2] J. Thompson, Finite groups with fixed-point-free automorphosms of prime order, Proc. Nat. Acad. Sci. U.S.A., 45 (1959), 578-581.

[3] B. Hartley A general Brauer-Fowler theorem and centralizers in locally finite groups, Pacific J. Math. 152 (1992), 101-117.

[4] G. Higman, Groups and rings which have automorphisms without non-trivial fixed elements, J. London Math. Soc. (2) 32 (1957), 321-334.

[5] V. A. Kreknin, The solubility of Lie algebras with regular automorphisms of finite period, Dokl. Akad. Nauk SSSR 150 (1963), 467-469; English transl., Math. USSR Doklady 4 (1963), 683-685.

[6] V.A. Kreknin and A. I. Kostrikin, Lie algebras with regular automorphisms, Dokl. Akad. Nauk SSSR 149 (1963), 249-251 (Russian); English transl., Math. USSR Doklady 4, 355-358.

[7] E. I. Khukhro, Groups and Lie rings admitting an almost regular automorphism of prime order, Mat. Sbornik 181, no. 9 (1990), 1207-1219; English transl., Math. USSR Sbornik 71, no. 9 (1992), 51-63.

[8] L. G. Kovács, Groups with regular automorphisms of order four, Math. Z. 75 (1960/61), 277-294.

[9] N. Yu. Makarenko and E. I. Khukhro, Finite groups with an almost regular automorphism of order four, Algebra Logika 45 (2006), 575-602; English transl., Algebra Logic 45 (2006), 326-343.

[10] J. L. Alperin, Automorphisms of solvable groups, Proc. Amer. Math. Soc. 13 (1962), 175-180.

[11] E. I. Khukhro, Finite $p$-groups admitting an automorphism of order $p$ with a small number of fixed points, Mat. Zametki 38 (1985), 652-657 (Russian); English transl., Math. Notes 38 (1986), 867-870.

[12] Shalev A. On almost fixed point free automorphisms, J. Algebra, 157, 271-282.

[13] Khukhro E. I. Finite $p$-groups admitting $p$-automorphisms with few fixed points, Mat. Sbornik, 184 (1993), 53-64; English transl., Russian Acad. Sci. Sbornik Math., 80 (1995), 435-444. 
[14] Yu. Medvedev p-Divided Lie rings and p-groups, J. London Math. Soc. (2), 59 (1999), 787-798.

[15] A. Jaikin-Zapirain, On almost regular automorphisms of finite p-groups, Adv. Math. 153 (2000), 391-402.

[16] J. Thompson, Automorphisms of solvable groups, J. Algebra 1 (1964), 259-267.

[17] A. Turull, Fitting height of groups and of fixed points, J. Algebra 86 (1984), 555-566.

[18] B. Hartley and I. M. Isaacs, On characters and fixed points of coprime operator groups, J. Algebra 131 (1990), 342-358.

[19] S. D. Bell, B. Hartley, A note on fixed-point-free actions of finite groups, Quart. J. Math. Oxford Ser. (2), 41 (1990), N 162, 127-130.

[20] E. C. Dade, Carter subgroups and Fitting heights of finite solvable groups, Illinois J. Math. 13 (1969), 449-514.

[21] G. Ercan, On a Fitting length conjecture without the coprimeness condition, Monatsh. Math. 167, no. 2 (2012), 175-187.

[22] G. Ercan and İ. Güloğlu, On finite groups admitting a fixed point free automorphism of order $p q r$, J. Group Theory 7, no. 4 (2004), 437-446.

[23] G. Ercan and İ. Güloğlu, Fixed point free action on groups of odd order, J. Algebra 320 (2008), 426-436.

[24] Unsolved Problems in Group Theory. The Kourovka Notebook, no. 18, Institute of Mathematics, Novosibirsk, 2014.

[25] B. Hartley and V. Turau, Finite soluble groups admitting an automorphism of prime power order with few fixed points, Math. Proc. Cambridge Philos. Soc., 102 (1987), 431-441.

[26] Rae, A. Sylow p-subgroups of finite p-soluble groups. J. London Math. Soc. (2) 7 (1973), $117-123$.

[27] Hartley, B.; Rae, A. Finite p-groups acting on $p$-soluble groups. Bull. London Math. Soc. 5 (1973), 197-198.

[28] Kurzweil, Hans. Eine Verallgemeinerung von fixpunktfreien Automorphismen endlicher Gruppen. (German) Arch. Math. (Basel) 22 (1971), 136-145.

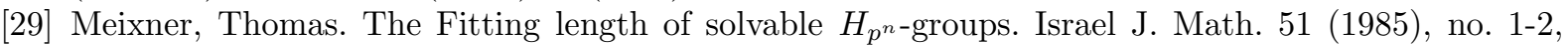
68-78

[30] Wilson, John S. On the structure of compact torsion groups. Monatsh. Math. 96 (1983), no. 1, 57-66.

[31] Khukhro, E. I.; Shumyatsky, P. Words and pronilpotent subgroups in profinite groups. J. Aust. Math. Soc. 97 (2014), no. 3, 343-364.

[32] P. Hall and G. Higman, The $p$-length of a $p$-soluble group and reduction theorems for Burnside's problem, Proc. London Math. Soc. (3), 6 (1956), 1-42.

[33] A. H. M. Hoare, A note on 2-soluble groups, J. London Math. Soc. 35 1960, 193-199.

[34] T. R. Berger and F. Gross, 2-length and the derived length of a Sylow 2-subgroup, Proc. London Math. Soc. (3) 34 (1977), 520-534.

[35] E. G. Bryukhanova, The relation between 2-length and derived length of a Sylow 2-subgroup of a finite soluble group, Mat. Zametki 29, no. 2 (1981), 161-170; English transl., Math. Notes 29, no. 1-2 (1981), 85-90.

[36] B. Hartley, Automorphisms of finite soluble groups. Preliminary version, MIMS EPrint: 2014.52 http://eprints.ma.man.ac.uk/2188/01/covered/MIMS_ep2014_52.pdf

[37] B. Hartley, Some theorems of Hall-Higman type for small primes, Proc. London Math. Soc. (3) 41 (1980), 340-362.

[38] C. W. Curtis and I. Reiner, Representation theory of finite groups and associative algebras, Interscience, New York-London, 1962.

[39] E. Khukhro and V. Mazurov, Automorphisms with centralizers of small rank, Groups St. Andrews 2005. Vol. II. Selected papers of the conference, St. Andrews, UK, July 30-August 6, 2005, London Math. Soc. Lecture Note Ser., vol. 340, Cambridge Univ. Press, Cambrisge, 2007, 564-585.

Sobolev Institute of Mathematics, Novosibirsk, Russia,

AND UNIVERSITY OF LINCOLN, UK

E-mail address: khukhro@yahoo.co.uk 